\title{
A Novel Approach for Pseudo-Haptic Textures Based on Curvature Information
}

\author{
Ferran Argelaguet, David Antonio Gómez Jáuregui, Maud Marchal and \\ Anatole Lécuyer \\ INRIA Rennes \\ \{fernando.argelaguet_sanz, david.gomez_jauregui, maud.marchal, \\ anatole.lecuyer\}@inria.fr
}

\begin{abstract}
Pseudo-haptic textures allow to optically-induce relief in textures without a haptic device by adjusting the speed of the mouse pointer according to the depth information encoded in the texture. In this work, we present a novel approach for using curvature information instead of relying on depth information. The curvature of the texture is encoded in a normal map which allows the computation of the curvature and local changes of orientation, according to the mouse position and direction. A user evaluation was conducted to compare the optically-induced haptic feedback of the curvature-based approach versus the original depth-based approach based on depth maps. Results showed that users, in addition to being able to efficiently recognize simulated bumps and holes with the curvature-based approach, were also able to discriminate shapes with lower frequency and amplitude.
\end{abstract}

\section{Introduction}

Pseudo-haptic feedback is a well-known interaction technique that allows to create an illusion of haptic properties with the combination of visual stimuli and passive input devices [7]. When introducing a decouple between the visual stimuli and the users' actions, the visual stimuli is dominant and can successfully create a haptic illusion. Several studies proved that pseudo-haptic feedback can be used to simulate various haptic sensations such as friction [7], the degree of hardness or softness of an object [5], the mass of a virtual object [2] or the relief of a $2 \mathrm{D}$ image [6]. Its main advantage is that it does not require any dedicated device to simulate haptic stimuli. For example, to simulate pseudo-haptic feedback in a desktop computer, a standard mouse is enough. Thus, the pseudo-haptic feedback can be used in a wide range of applications (e.g. 2D GUIs, video games, virtual reality, tactile images).

In this paper, we focus on the improvement of pseudo-haptic textures [6]. Pseudo-haptic textures allow to simulate the relief of a $2 \mathrm{D}$ texture by adjusting the control-display ratio of the mouse cursor. As the cursor advances along the texture, the CD ratio is adjusted in a per-pixel basis according to the information stored in a depth map. Unfortunately, according to haptic studies [11], [12], using only depth information does not provide a strong cue for shape discrimination. 
In addition, it is difficult to balance the effect of the depth-based approach for high depth ranges.

In this work, we introduce the use of curvature information to enhance the illusion of the pseudo-haptic textures. The curvature is encoded into a normal map, which provides information about the curvature at each pixel and determines changes of the local orientation of the surface. Normal maps are commonly used in computer graphics and haptics to store high frequency data, they can be computed procedurally or using a 3D graphic editing software. Although the curvature information of a surface is an important discriminant for haptic perception, as far as we know, there is no study which explores its integration with pseudo-haptic textures. We believe that the curvature information can provide a stronger cue about the shape of textures that using only depth information.

The remaining of the paper is structured as follows. In Section 2, we discuss previous work on pseudo-haptic textures and haptic perception using curvature information. In Section 3, we present the proposed algorithm based on curvature information. Next, in Section 4, we detail and discuss the results of the user evaluation comparing our approach with the depth-based approach. Finally, Section 5 provides concluding remarks and future work.

\section{Related Work}

\subsection{Pseudo-Haptic Textures Based on Depth Maps}

Several works have studied the efficiency of pseudo-haptic effects in order to simulate haptic properties. As one of the first experiments, Lécuyer et al. [7] simulated friction with a virtual cube moved by the user using a $2 \mathrm{D}$ mouse and a Spaceball. In this experiment, the virtual cube was decelerated when crossing a gray area by altering artificially the Control/Display ratio (Control refers to the speed of hand movement while Display refers to the speed of cursor movement). Here, users were able to perceive "friction" with an increased C/D ratio without using a haptic device.

In a posterior study, the same authors [6] investigated the simulation of tactile sensations on 2D textures using a pseudo-haptic approach. The main idea consisted in controlling the speed of the mouse cursor as a function of the depth information of the texture over which the mouse cursor was traveling. For example, when the mouse cursor was moving along a surface with a positive slope, the speed of the cursor was decreased; on the opposite case, a negative slope produced an acceleration of the mouse cursor. In these experiments, the users were able to efficiently identify simulated bumps and holes in the texture. Hachisu et al. [4] also used the depth information of the textures to combine the pseudo-haptic effect with visual and tactile vibrations in order to strengthen the tactile perception.

Mensvoort et al. [8] compared the usability of mechanically simulated haptic textures with optically simulated haptic textures. The authors used the slope vector of the texture to increase or decrease the speed of the mouse cursor. In 
order to compute the slope, they used the depth map of the texture as source of information. Later, the same authors [9] compared the perceptual differences of recognizing bumps and holes using pseudo-haptic textures with the simulation generated by a mechanical force feedback device. The results showed that in some cases, for example, for subtle forces, optically simulated haptic feedback can be even more expressive than mechanical simulations of force feedback.

\subsection{Haptic Perception Using Curvature Information}

Although previous work present important improvements and applications of pseudo-haptic textures, there is no study that investigates the curvature information of the texture in order to enhance the pseudo-haptic effect. Several prior studies using haptic devices have shown that the orientation of the surface of contact is a dominant source of information for perceiving shape [3]. Bernard et al. [10] presented an experiment in which users had to distinguish an elliptical cylinder from a circular cylinder using a haptic stimuli. In the elliptical cylinder, the local curvature varies over the surface, whereas it is constant for the circular cylinder. The results showed that an ellipse can be distinguished from a circle when divergences or changes in curvature can be perceived. In contrast, when curvature information was lacking, shape recognition performance decreased.

Wijntjes et al. [11] investigated the specific contribution between the curvature (local orientation) and depth cues in order to perceive the shape of curved surfaces. Their results demonstrated that discrimination performances depend largely on the availability of local orientation. They also found that a curved shape that is defined solely by a height profile is hard to discriminate. In a more recent study, Zeng et al. [12] constructed a shape-simulating haptic device that depends totally on the curvature information. The haptic device was able to move and rotate a flat plate. The rotation angle was dependent on the position of the contact point and it varied following the position of fingertip in order to satisfy the local orientation of the curved surface. The results verified that users were able to efficiently perceive distinct curved shapes.

\section{Pseudo-Haptic Textures Based on Curvature}

According to the results showed in haptic studies for curvature perception, we believe that the curvature and local changes of orientation can be exploited in order to enhance the exploration of a pseudo-haptic texture. While the original approach [6] only uses depth information to modify the Control/Display ratio, our proposed method relies on the curvature information of the surface encoded into a normal map. When the cursor moves from one pixel to another, the CD ratio is computed according to the curvature at the midpoint of both pixels and to the changes of the local orientation. Changes of the $\mathrm{CD}$ ratio can be seen as the effect of lateral forces [1]. In the following, we describe the details of our proposed approach that integrates the curvature information with the local changes of orientation as important cues for simulating pseudo-haptic textures. The algorithm differs from [6] mainly for the computation of the CD ratio. 


\subsection{Algorithm Description}

Input. Assuming that the interactive area is restricted to the pseudo-haptic texture, the algorithm has as inputs (see Figure 1 Left): (a) the normal map $\left(M_{n}\right)$, (b) the current mouse cursor position $p_{m}=\left(x_{m}, y_{m}\right)$, (c) the direction of the mouse $\mathbf{m}$ and (d) the physical distance covered by the mouse $d_{m}$.

Overview. According to the input variables, the algorithm updates the position of mouse cursor (starting at $p_{m}$ ) by traversing the texture along the direction defined by $\mathbf{m}$. Each time the mouse cursor advances one pixel, a new $\mathrm{CD}$ ratio is computed according to the current and previous pixel. The traversal finishes when the accumulated distance is equal or greater than $d_{m}$. Additional details can be found in $[6]$.

Curvature computation. In order to compute the CD ratio, we need to retrieve the curvature information between pixels. Let consider two neighboring pixels $p_{1}=\left(x_{1}, y_{1}, 0\right)$ and $p_{2}=\left(x_{2}, y_{2}, 0\right)$ and their respective normals $\mathbf{n}_{1}$ and $\mathbf{n}_{\mathbf{2}}$ (see Figure 1 Middle). We define the plane $\pi$ considering the direction of the mouse $\mathbf{m}$, the viewing direction $\mathbf{v}=(0,0,-1)$ and $p_{1}$. Then, we apply an affine transformation to $\pi$, transforming it into $x y$ plane (referred as $\pi^{\prime}$ ); the same transformation is applied to vectors $\mathbf{n}_{\mathbf{1}}$ and $\mathbf{n}_{\mathbf{2}}$ obtaining $\mathbf{n}_{\mathbf{1}}^{\prime}$ and $\mathbf{n}_{\mathbf{2}}^{\prime}$. Finally, we project the normals $\mathbf{n}_{\mathbf{1}}^{\prime}$ and $\mathbf{n}_{\mathbf{2}}^{\prime}$ into the plane $\pi^{\prime}$ obtaining $\mathbf{n}_{\mathbf{1}}^{\prime \prime}$ and $\mathbf{n}_{\mathbf{2}}^{\prime \prime}$ (see Figure 1 Right). Notice that the $x$-axis relates to the direction of movement, while the negative $y$-axis relates to the viewing direction. From $\mathbf{n}_{\mathbf{1}}^{\prime \prime}$ and $\mathbf{n}_{\mathbf{2}}^{\prime \prime}$, and using Equation 1, we can obtain the curvature represented by $\alpha$ and the local change of curvature $(\Delta \beta)$.

$$
\begin{gathered}
\cos (\alpha)=\left(n_{1 x}^{\prime \prime}+n_{2 x}^{\prime \prime}\right) / 2 \\
\Delta \beta=\arccos \left(n_{1 x}^{\prime \prime}\right)-\arccos \left(n_{2 x}^{\prime \prime}\right)
\end{gathered}
$$

The cosine of $\alpha$ determines the magnitude of the slope and its sign and $\Delta \beta$ provides information about the change of the local orientation. If $\Delta \beta>0$, the slope is locally increasing and vice-versa.
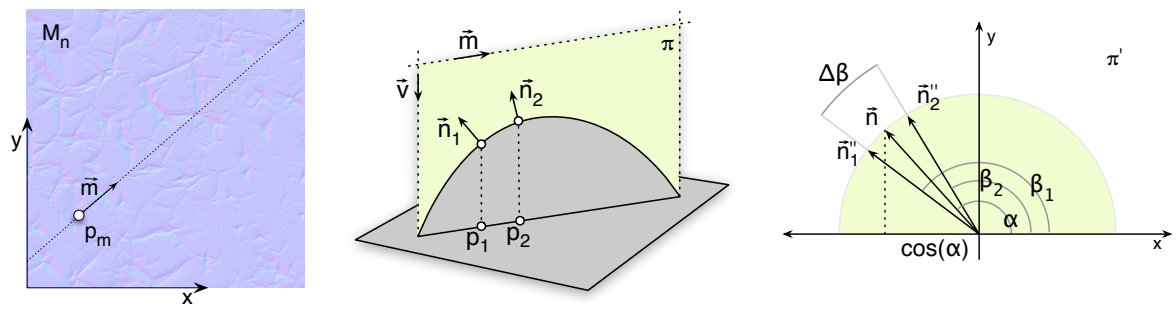

Fig. 1: Left, top view of the pseudo-haptic texture. Middle, the computation of the $\mathrm{CD}$ ratio only takes into account the projection of the normals into the plane $\pi$. Right, relationship of the normals of the two pixels with $\alpha$ and $\beta$. 
CD Ratio computation. The computation of the CD ratio has two components, the influence of the $\cos (\alpha)$ and the influence of $\Delta \beta$. First, the $\cos (\alpha)$ is considered as a lateral force which models the friction of the surface. If it is negative, it applies a force against the movement and vice-versa. As the cosine is normalized between -1 and 1 we need to apply two scaling constants determining the maximum and minimum $\mathrm{CD}$ ratio. Considering that a $\mathrm{CD}$ ratio of 1 refers to a flat surface $(\cos (\alpha)=0)$, the $\mathrm{CD}$ ratio is modeled as shown in Equation 2. $k_{\min }$ and $k_{\max }$ are user defined constants determining the maximum and minimum CD ratio.

$$
\mathrm{CD} \text { ratio }= \begin{cases}1-\cos (\alpha) \cdot\left(1-1 / k_{\min }\right) & \text { if } \cos (\alpha)>0, k_{\min } \geq 1 \\ 1-\cos (\alpha) \cdot\left(k_{\max }-1\right) & \text { if } \cos (\alpha)<0, k_{\max } \geq 1\end{cases}
$$

However, this formulation accounts for the local curvature but not for changes in curvature. The same value of $\cos (\alpha)$ may result from different values of $\mathbf{n}_{\mathbf{1}}$ and $\mathbf{n}_{\mathbf{2}}$. As the computation of the CD ratio is done for each pair of pixels, without considering previous pixels, it would produce unnoticeable effects for sharp surfaces and edges. For that purpose, the factors $k_{\max }$ and $k_{\min }$ were adjusted according to $\Delta \beta$ (see Equation 3). $\Delta \beta$ has its units in degrees. If $\Delta \beta \approx$ 0 , the CD ratio only accounts for $\alpha$. Now, $k_{\text {max }}^{\prime}$ and $k_{\text {min }}^{\prime}$ determine the maximum and minimum CD ratio when $\Delta \beta=0$. In order to avoid extreme values of $k_{\min }$ and $k_{\max }$, a lower and upper limit can be defined.

$$
\begin{aligned}
& k_{\text {max }}=k_{\text {max }}^{\prime} \cdot(1+\Delta \beta) \\
& k_{\text {min }}=k_{\text {min }}^{\prime} \cdot(1-\Delta \beta)
\end{aligned}
$$

Figure 2 shows the behavior of the $\mathrm{CD}$ ratio according to $\alpha$ and $\Delta \beta$. The plot only considers smooth variations of curvature $(\Delta \beta= \pm 2)$.

\subsection{Comparison with the Depth-Based Approach}

Both approaches exhibit a similar behavior, crossing two pixels with a positive slope will require a CD ratio greater than 1 , and vice-versa. However, the CD ratio for the depth-based approach is obtained taking into account depth changes between pixels $(\Delta h)$. Changes of $\mathrm{CD}$ ratio exhibit a linear behavior according to $\Delta h$. Similar to our approach, it also requires to determine the maximum and minimum CD ratio, but as the depth map is typically normalized between 0 and 1 , we also need to provide a scaling factor for the depth map.

In contrast to the linear behavior of $\Delta h$, the behavior of $\cos (\alpha)$ is not linear. It provides higher variation for values of $\alpha>3 \pi / 4$ and $\alpha<\pi / 4(\alpha \in[0 . . \pi])$. This provides higher precision for surfaces with smaller slopes, thus being easier for the user to identify them.

Another difference is that our approach is able to simulate $C^{0}$ discontinuities. One of the results detailed in [6] showed that users tend to recognize sharp surfaces as smooth surfaces. As we consider local variations of curvature $(\Delta \beta)$, non-continuous changes in curvature result in stronger variations of the $\mathrm{CD}$ ratio, which now will be noticeable for the user. All these differences are expected to provide better shape recognition. 

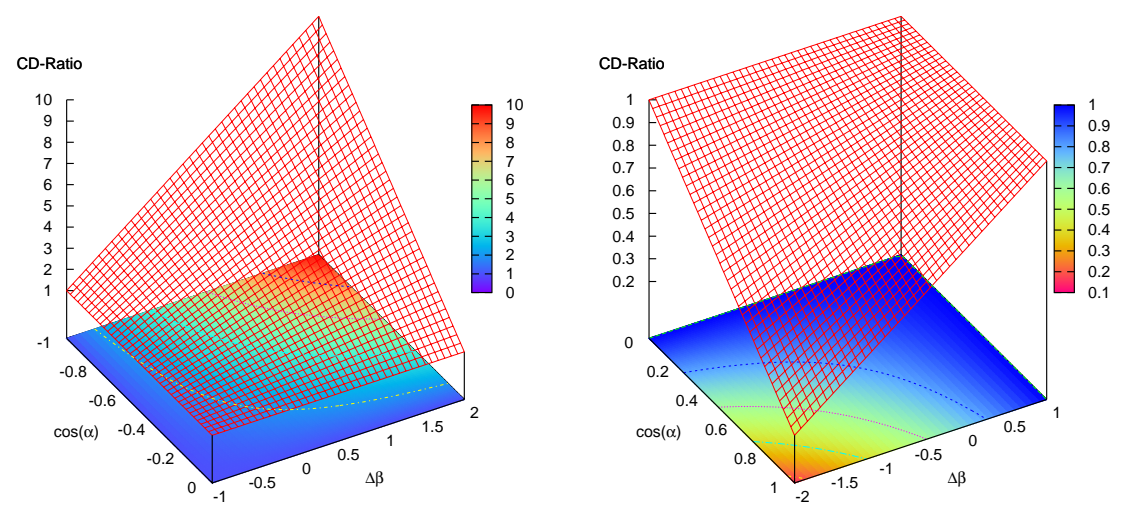

Fig. 2: CD-ratio variation as a function of $\Delta \beta$ and $\cos (\alpha)$. The graph on the left depicts it variation for positive slopes and the right for negative slopes. The upper and lower limit for $k_{\max }$ and $k_{\min }$ were 30 and 5 , and 4 and 1.5 for $k_{\max }^{\prime}$ and $k_{\text {min }}^{\prime}$.

\section{User Evaluation}

The analysis of the depth-based approach has revealed existing limitations when wiling to recognize pseudo-haptic textures with varying depth ranges. In this user evaluation we want to explore the limits of the depth-based approach when trying to recognize shapes with different depth ranges and whether the curvature-based approach is able to provide better results. For this purpose we have conducted a similar experiment to [6], based on the recognition of simple targets with different shapes and profiles.

\subsection{Procedure}

The visual stimuli was a rectangular area shaded with a constant dark blue of $500 \mathrm{px}$, placed at the center of the screen. Users were presented with a simple task: identify if the shape placed inside was a bump or a hole. They were instructed to perform horizontal mouse movements along the boundaries of the rectangular area. The only feedback provided was the speed of the mouse cursor, which was adjusted according to the pseudo-haptic technique being evaluated. For each shape, after ten seconds of exploration an answer screen was displayed, asking the user to classify the shape. Users could only choose the option bump or hole, they were instructed to choose a random option in case of not being able to recognize the shape. Also, in order to explore if users were able to recognize the shape in less time, they could press the left mouse button to display the answer screen. Once the user chooses an option, the next target is "displayed". This process was repeated until the end of the experiment. 


\subsection{Design and Hypothesis}

Twenty four different targets were considered in the experiment (see Figure 3). They were built according to the shape (Bump or Hole), the simulated profile (Linear, Gaussian and Polynomial), its height (0.25 and 1, the depth map was normalized between -1 and 1) and two different radius (100px and 200px).

According to the targets' configuration and the techniques evaluated, the independent variables were the pseudo-haptic technique (Depth and Curvature), the Shape, the Profile, the Heights (High, Low) and the Radius (Large, Small) of the targets, all considered as whithin-subjects. Thus resulting in a $2 \times 2 \times 3 \times 2 \times 2$ factorial design (48 combinations). The dependent variables were the number of misclassified shapes and the time to complete the task. For the task-completiontime, we only considered the time spent during the active exploration of the shape.

The experiment was subdivided into two phases, one for each Technique. Each phase was also subdivided into three blocks. For each block, users had to classify the 24 targets which were ordered randomly. For each phase, the first block was considered as training/adaptation and was not considered in the analysis. Participants required in average twenty minutes to finish the experiment and they could take a break at the end of the first phase.

Our main hypotheses were that users will significantly misclassify less shapes when using the curvature-based approach (H1.1), with higher differences for targets with a smaller depth range (H1.2). Regarding recognition time, we also expect lower mean of recognition time for the curvature-based approach (H2.1). In addition, we also expect a lower mean for the recognition time for targets with higher depth range (H2.2).

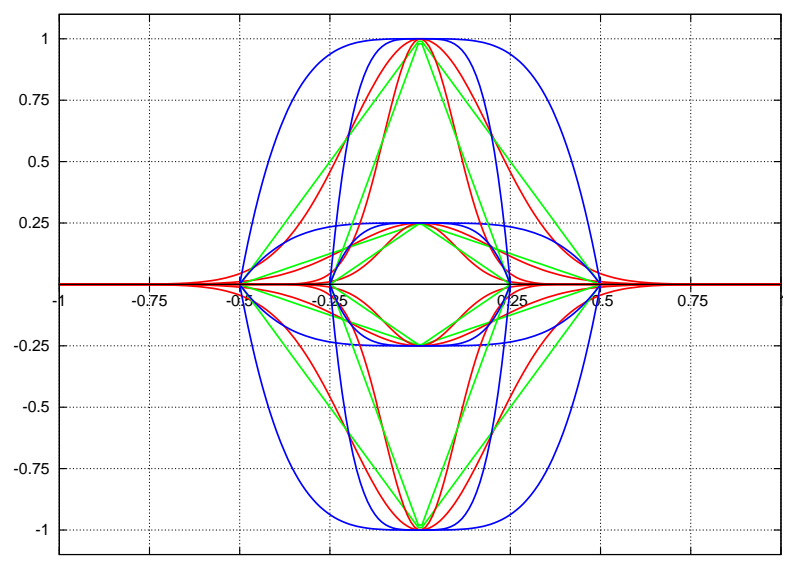

Fig. 3: Shapes used during the experiment. Linear (green), Polynomial (blue) and Gaussian (red). The Equation for the polynomial profile was $f(x)=1-x^{4}$. 


\subsection{Experimental Apparatus and Population}

The experiments were conducted in a 24" inch monitor with a resolution of $1920 \times$ 1200 pixels, using as input device a standard mouse. The mouse acceleration provided by the operative system was disabled. Participants were placed at 60 $\mathrm{cm}$ from the screen and operated the mouse with their dominant hand.

Twelve volunteer users (undergraduate and graduate students) took part in the experiment, aged from 23 to 31, 2 females and 11 males. All users had not experienced pseudo-haptic feedback before.

\subsection{Results}

For the statistical analysis we used ANOVA analysis. For all post-hoc comparisons, Bonferroni adjustments for $\alpha=95 \%$ were applied; only significant post-hoc comparisons are mentioned $(p<0.05)$.

Error rate. Regarding the number of misclassified targets versus the Technique, Radius, Height, Profile and Shape, there were two main significant effects for Technique $\left(F_{1,11}=8.11, p<0.01\right)$ and for Height $\left(F_{1,11}=18.78, p<\right.$ 0.001 ). Post-hoc tests showed that users did more mistakes when employing the depth-based approach $(7.47 \%)$ in comparison with the curvature-based approach (3.47\%), thus accepting H1.1. Post-hoc tests also showed that users had more difficulties to classify targets with lower height, which also supports H1.2. No main effects were found for shape, radius and profile.

The two-way interactions found significant were Radius and Height $\left(F_{1,11}=\right.$ $6.76, p<0.01)$, Technique and Height $\left(F_{1,11}=5.42, p<0.05\right)$, Technique and Radius $\left(F_{1,11}=6.64, p<0.01\right)$. The ANOVA also showed a three-way significant interaction between Technique, Radius and Height $\left(F_{1,11}=9.58, p<0.005\right)$. Post-hoc tests showed that users performed worst when combining large radius with lower heights, however a deeper analysis, showed that it only happened when the users were recognizing the shapes with the depth-based approach. The effect was non-significant for the curvature-based approach.

Recognition Time. The ANOVA showed main effects for Technique $\left(F_{1,11}=\right.$ $101.13, p<0.001)$, Radius $\left(F_{1,11}=14.99, p<0.001\right)$ and Height $\left(F_{1}=276.88, p<\right.$ $0.001)$. Post-hoc tests showed that users were able to recognize the targets faster with the curvature-based approach (6.03s) than with the depth approach (4.88s), thus accepting H2.1. In addition, users required significantly more time to recognize targets with lower height and larger radius, which also supports H2.2.

The ANOVA also showed two-way interactions and three-way interaction for Technique, Radius and Height: Radius and Height $\left(F_{1,11}=18.52, p<0.001\right)$, Technique and Radius $\left(F_{1,11}=16.15, p<0.001\right)$, Technique and Height $\left(F_{1,11}=\right.$ $68.53, p<0.001)$, and Technique, Radius and Height $\left(F_{1,11}=11.64, p<0.001\right)$. Post-hoc tests showed that the combination of low height and large radius resulted in the targets which required more exploration time (see Figure 4), specially for the depth approach $(7.9 \mathrm{~s})$ than for the curvature-based approach (5.4s). 


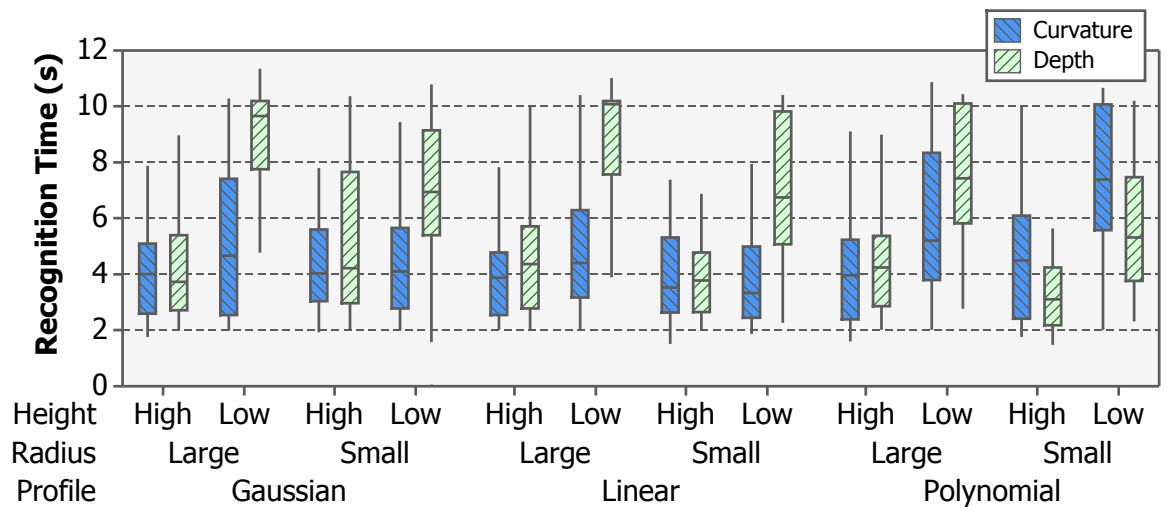

Fig. 4: Boxplot of the mean recognition time for each Technique, grouped by Profile, Radius and Height.

There was also a two-way interaction effect between Technique and Profile $\left(F_{2,11}=28.23, p<0.001\right)$ and a three-way interaction between Technique, Profile and Height $\left(F_{2,11}=13.68, p<0.001\right)$. Post-hoc tests showed that users significantly needed more time to recognize the shape using the Depth-based approach for Gaussian and Linear profiles, specially for targets with lower height. In contrast, for the curvature-based approach users required significantly more time to recognize Polynomial profiles (see Figure 4).

\subsection{Discussion}

From the results, we can state that both techniques presented a similar behavior in terms of shape recognition except for the combination of large radius and low height. For these targets, users made significantly more errors and required more time to recognize them with the depth-based approach.

At the end of the experiment, most users reported that they focused more in the deceleration of the mouse cursor rather than in the acceleration to determine the shape. Users explained the following strategy in order to locate the shapes: if the mouse slows down in the first half of the shape it is classified as a bump and if it slows down on the second half of the shape it is classified as a hole.

Figure 5 depicts the variation of the CD-ratio when the mouse cursor moves horizontally from the left side of the target to the right size. Targets with large radius and lower height presented the minimum $\mathrm{CD}$ ratio range. Being the $\mathrm{CD}$ ratio for the depth-based approach the lowest. This low $\mathrm{CD}$ ratio range resulted in less perceptible mouse speed changes which made the recognition of the targets more difficult. In contrast, for the curvature-based approach the $\mathrm{CD}$ ratio ranges were higher (see Figure 5, Left). That is our main explanation for users being able to easily recognize targets with the curvature-approach. Although we could have increased the acceleration and deceleration effect for the depth-based 
approach it would have increased too much the CD-ratio range for the other profiles. Acceleration and deceleration factors were adjusted taking into account the targets having higher depth ranges. Figures $5 \mathrm{~b}$ and $5 \mathrm{~d}$ depict the CD ratio range for targets with higher slope.

However, the curvature-based approach poses two main limitations. The first limitation is related to non-continuous surfaces. Our method is not be able to recognize a discontinuity and will proceed as if the surface is continuous, which

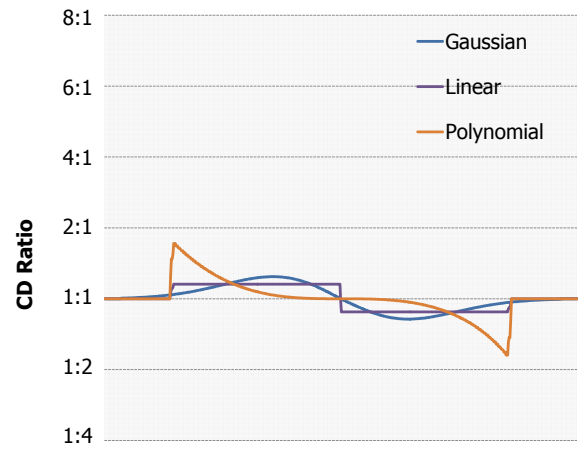

(a) Depth-based

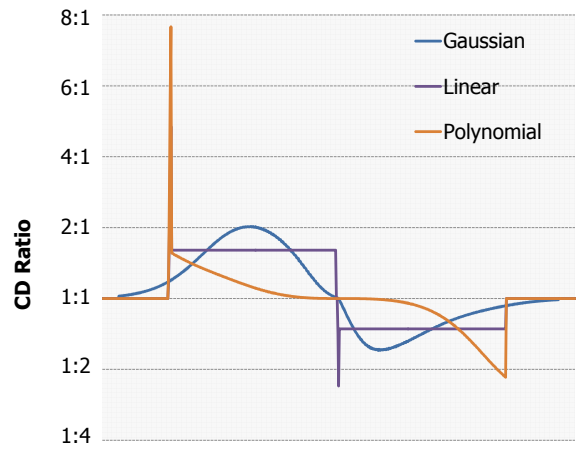

(c) Curvature-based

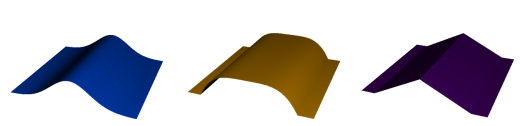

(e) Profiles

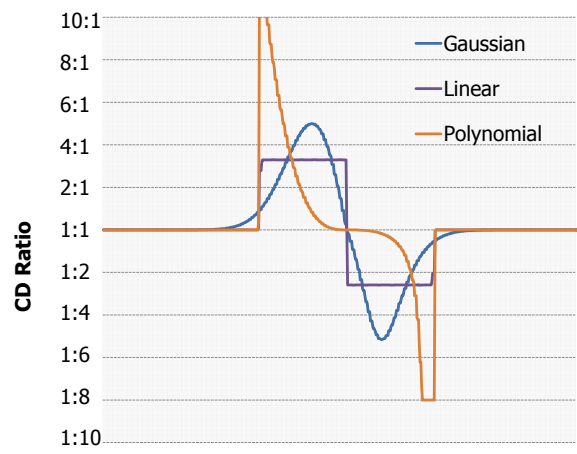

(b) Depth-based

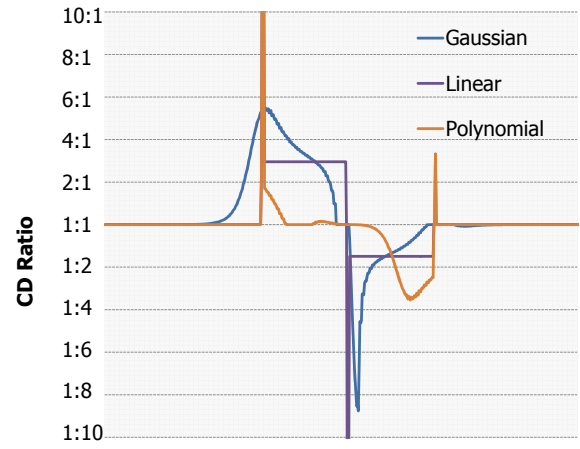

(d) Curvature-based

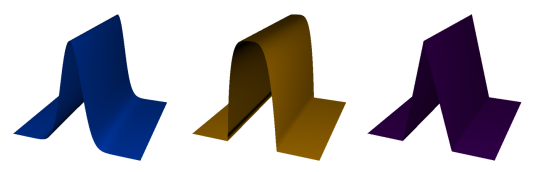

(f) Profiles

Fig. 5: Evolution of the CD ratio while moving the mouse from the leftmost of the target to the rightmost (bump shapes). (a,c), targets with large radius and lower height. (b,d), targets with small radius and higher height. (e,f), 3D representation of the simulated profiles. 
might result in an unexpected behavior for the user. A possible solution would be to detect the discontinuities using depth information and apply the depth-based approach for this situation. The second limitation is high frequency surfaces. While the depth-based approach will not be able to detect them, the curvature in this surfaces will change abruptly, resulting in rapid changes of the CD ratio. This effect can also result as an unexpected behavior for the user. A possible course of action can be focused on use depth information to smooth the normal map locally, for instance keep high frequency surfaces close to the viewer and smooth those further away.

On the other hand, one advantage of our approach is that it contains the information provided by the depth-based approach, from the slope of the surface we can compute the difference of depth between two pixels. In addition, it dos not require the usage of a scaling factor. Furthermore, our approach is also able to account for abrupt changes of curvature. For example, in the Linear and Polynomial profiles, when the cursor cross a discontinuity $\left(C^{0}\right.$ surface $)$, due to the abrupt change of curvature a strong change of the $\mathrm{CD}$ ratio is provided.

\section{Conclusion}

Focusing in the limitation of depth for pseudo-haptic textures and the fact that the curvature information of the surface is considered as an important haptic cue, we have proposed a novel pseudo-haptic approach that takes into account the local orientation of the surface and how it varies. The Control/Display ratio is computed as a function of the slope of the surface and the changes of the local orientation.

An experimental study was conducted in order to compare the proposed curvature-based approach versus the original approach based on depth maps. The study consisted in evaluating the recognition of bumps and holes with different heights, radius and profiles. The only feedback provided was the variation of the speed of the mouse cursor. The results showed that participants made
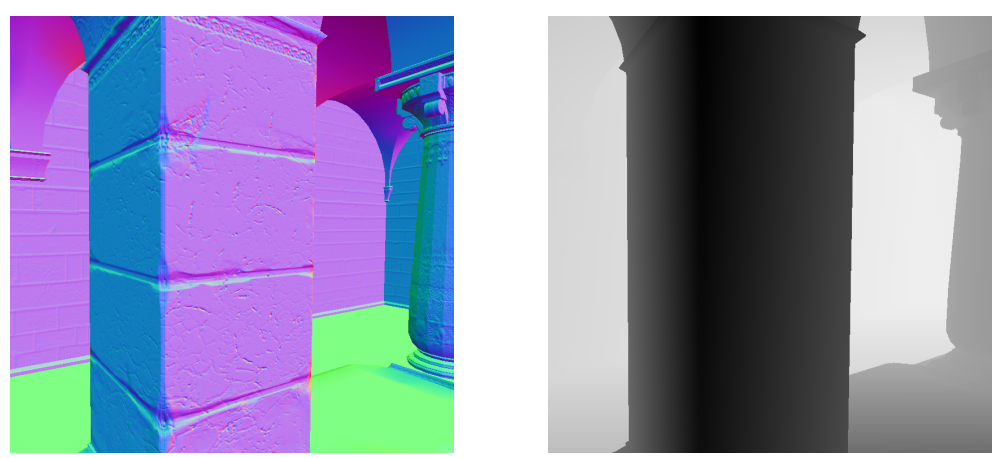

Fig. 6: Comparison of the normal map (left) and the depth map (right) of a 3D environment. Subtle details in the depth map are no longer detectable due to limited spatial resolution. The detail in the normal map is preserved. 
less number of mistakes when employing the curvature-based approach in comparison with the depth-based approach. Particularly, a significantly less number of errors was obtained for shapes of large radius and low height. Participants also required in overall more time to recognize the shape of the targets with the depth-based approach.

As a future work, we plan to explore whether the curvature-based approach allows to provide better shape recognition for more complex shapes and surfaces and we also plan to extend the proposed approach to computer generated 3D environments (see Figure 6) as well as some other potential applications like surface analysis, medical simulators, navigation in 3D websites or video games.

\section{References}

1. G. R. de-la Torre and V. Hayward. Force can overcome object geometry in the perception of shape through active touc. In Nature 412, pages 445-448, 2001.

2. L. Dominjon, A. Lécuyer, J. M. Burkhardt, P. Richard, and S. Richir. Influence of control/display ratio on the perception of mass of manipulated objects in virtual environments. In Proceedings of the IEEE International Conference on Virtual Reality, pages 19-25, 2005.

3. I. Gordon and V. Morison. The haptic perception of curvature. In Perception and Psychophysics, volume 31, pages 446-450, 1982.

4. T. Hachisu, G. Cirio, M. Marchal, A. Lécuyer, and H. Kajimoto. Pseudo-haptic feedback augmented with visual and tactile vibrations. In Proceedings of International Symposium on VR Innovations (ISVRI'11), pages 331-332, 2011.

5. A. Lécuyer. Simulating haptic feedback using vision: A survey of research and applications of pseudo-haptic feedback. In Presence: Teleoperators and Virtual Environments, volume 18, pages 39-53. MIT Press Cambridge, MA, USA, 2009.

6. A. Lécuyer, J.-M. Burkhardt, and L. Etienne. Feeling bumps and holes without a haptic interface: the perception of pseudo-haptic textures. In Proceedings of the SIGCHI conference on Human factors in computing systems, CHI '04, pages 239-246. ACM, 2004.

7. A. Lécuyer, S. Coquillard, A. Kheddar, P. Richard, and P. Coiffet. Pseudohaptic feedback: can isometric input devices simulate force feedback? In IEEE International Conference on Virtual Reality (IEEE VR), pages 83-90, 2000.

8. K. v. Mensvoort, D. J. Hermes, and M. V. Monfort. Usability of visually simulated force feedback. International Journal of Human-Computer Studies, 66(6):438-451, 2008.

9. K. v. Mensvoort, P. Vos, D. Hermes, and R. v. Liere. Perception of mechanically and optically simulated bumps and holes. ACM Transactions on Applied Perception, 7(2):1-24, 2010.

10. B. J. van der Horst and A. M. L. Kappers. Using curvature information in haptic shape perception of 3d objects. Experimental Brain Research, 190(3):361-367, 2008.

11. M. W. A. Wijntjes, A. Sato, V. Hayward, and A. M. L. Kappers. Local surface orientation dominates haptic curvature discrimination. IEEE Transactions on Haptics, 2(2):94-102, 2009.

12. T. Zeng, F. Giraud, B. Lemaire-Semail, and M. Amberg. Haptic perception of curvature through active touch. In IEEE World Haptics Conference (WHC), pages 533-538, 2011. 